\title{
Pi 2 Micropulsations as Indicators of Substorm Onsets and Intensifications
}

\author{
Gordon RostokeR and John V. OlsoN \\ Institute of Earth and Planetary Physics and Department of Physics, \\ University of Alberta, Edmonton, Canada
}

(Received October 17, 1977)

\begin{abstract}
Pi 2 micropulsations are impulsive wave trains which accompany the onset of magnetospheric substorm disturbances. At middle and low latitudes Pi 2's are observed as relatively monochromatic damped wave trains, while at auroral observatories onsets of substorms are marked by impulsive increases in noise across the Pi 1 and Pi 2 bands. Because they are often clearly apparent on normal low latitude magnetograms their presence or absence has been used as an indicator of the presence or absence of substorm activity. In this paper we shall demonstrate the relationship between Pi 2 bursts and substorm onsets and intensifications. We shall show that the degree of spatial localization of the Pi 2 disturbance often makes it unidentifiable at middle and lower latitudes using conventional recording techniques. Thus while the presence of a $\mathrm{Pi} 2$ pulsation may be taken as an indication of substorm occurrence, its absence at a particular station may be due to the location of the station relative to the substorm region and may not necessarily imply the absence of substorm activity. Sonograms from an east-west array of observatories in the auroral oval will be employed to demonstrate the use of Pi signals at auroral zone latitudes to define the times of substorm onsets and intensifications. It is recommended that the present subjective means of identifying Pi 2's be replaced by more objective criteria, based upon careful analysis of Pi 2 signals.
\end{abstract}

\section{Introduction}

Since the pioneering work of Angenheister (1912), a great deal of attention has been paid to the morphology of impulsive damped wave trains recorded using conventional magnetographs (which measure absolute values of the main field) and induction coil magnetographs (which measure the rate of change of the magnetic field). These wave trains were initially categorized as sudden impulses (si) and sudden commencements (ssc) and later as pt whose higher frequency component was labelled spt. Eventually, in accordance with the recommendations of JACOBS et al. (1964), impulsive micropulsations were given a classification which characterized only their period range, namely $\mathrm{Pi} 1(1<T<40 \mathrm{sec})$ and $\mathrm{Pi} 2(40<T<150 \mathrm{sec})$. More recently a third category Pi $3(T>150 \mathrm{sec})$ has been suggested (SAITo, 1976) to cover extremely low frequency oscillations detected in substorm disturbed regions. SAIto and Yumoto (1978) have also introduced the term Ps 6 to cover very low frequency periodic changes in the auroral zone magnetic field during substorm activity. The boundaries of the 
frequency bands discussed above were not based upon any physical property of the pulsation other than frequency and there was no distinction made pertaining to generation mechanisms (Jacobs, private communication). Thus there is, at the present time, no reason to distinguish between low frequency Pi 1 and high frequency Pi 2 activity, for example.

For many years there was a tendency to study Pi activity as a subject area distinct from the study of substorms, even though it was realized (SAIto, 1961) that each Pi 2 observed at low latitudes signaled the occurrence of a magnetic bay at auroral zone latitudes. It was not until AKASOFU (1968) accumulated the work done using various research tools (e.g., magnetometers, riometers, all-sky cameras, etc.) under the umbrella of the magnetospheric substorm that it became customary to use any one of the many tools to infer substorm morphology. RosToker (1968) was one of the earliest workers to make extensive use of the Pi 2 as an indicator of substorm onsets. He found that there was normally a minimum of two Pi 2 wave trains associated with each substorm disturbance, and that a substaniial number of substorms were accompanied by more than two wave trains. He suggested, at that time, that the development of a substorm was a two stage process consisting of a trigger bay (the substorm onset) and the main bay (the development of the westward travelling surge) as shown in Fig. 1. FUKUnishi and Hirasaw A (1970) also noted that there were often several distinctive Pi 2 wave trains associated with a substorm, and they found the wave forms and power spectra of each wave train to be quite similar to one another.

Subsequently it became apparent that substorms developed as an organized series of impulsive electrojet intensifications, each electrojet element lying to the north or north-west of the pre-existing electrojet elements (Sergeev, 1974; Wiens and Rostoker, 1975). Each electrojet element has, associated with its onset, a Pi 2 disturbance which has a source region confined to the region of that electrojet element (OLson and Rostoker, 1975) suggesting that electrojets elements with a small scale size may have an associated Pi 2 which may only be observable close to the electrojet itself.

Since each electrojet element normally has an azimuthal extent of approximately $1 \mathrm{hr}$ in local time, or a time zone, the observations of the two stage character of substorms by Rostoker (1968) may then be understood. Any middle or low latitude observatory may normally detect Pi 2's which originate in its local time sector, viz. it will normally record visually identifiable Pi 2's for substorm onsets occurring up to one time zone on either side of the meridian of the observing station. Any Pi 2 onsets occurring more than one time zone away will not be identifiable due to low amplitudes. Thus for a sequence of several electrojet intensifications each one being to the northwest of the previous one, a normal mid-latitude observatory will detect, on the average, two Pi 2 onsets depending on the strength of the intensifications. It will generally be unable to detect onsets more than one time zone to the east or to the west under normal circumstances. Thus a given mid-latitude observatory may be unable to detect the actual onset times of a sequence of substorm intensifica- 

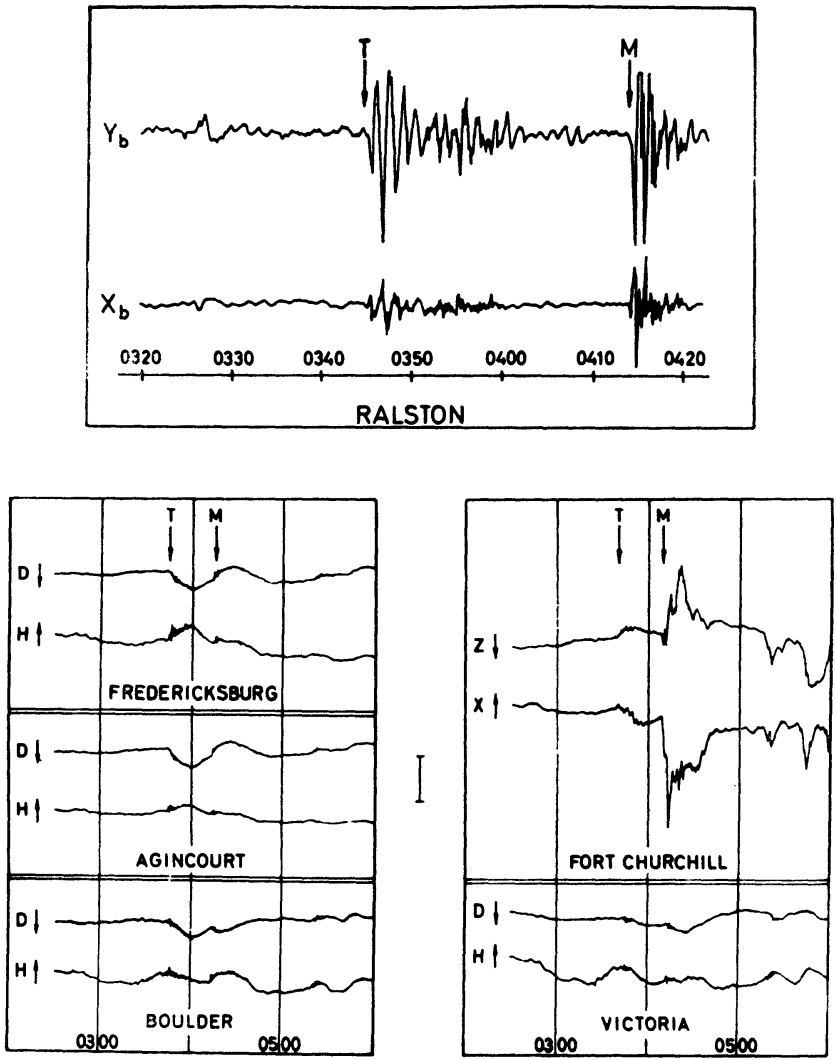

Fig. 1. Example of the structure of geomagnetic bays associated with a polar magnetic substorm and the accompanying Pi 2's recorded on June 6, 1965 (after RosTOKER, 1968). The top panel shows induction coil magnetograms from the mid-latitude station of Ralston and corresponding mid-latitude (Fredricksburg, Agincourt, Boulder and Victoria) magnetograms and an auroral zone (Fort Churchill) magnetogram are shown in lower panels. The Pi 2's are normally most easily identified in the horizontal perturbation fields. The Pi 2 activity in the auroral zone is masked by the steep ramp of the bay at auroral zone latitudes. The wave form at mid-latitude stations varies from site to site, and weaker Pi 2's may only appear as a thickening of the trace in midlatitude observatories.

tions if the onset takes place more than one time zone to the east. It may also fail to detect the end of the substorm sequence if the final electrojet element intensifies more than one time zone to the west. In essence, therefore, unless there is an adequate local time coverage of middle and low latitude magnetometers it may not be possible to identify the actual onset of an episode of substorm activity using Pi 2 pulsations. Furthermore, an absence of identifiable Pi 2 activity at middle and low latitude observatories does not rule out the existence of substorm activity given the present observatory distribution.

In the light of the recent trend towards utilization of Pi 2's as indicators of substorm onsets (Saito et al., 1976 a, b; Sakurai and Saito, 1976; Pytte et al., 
1978) we shall try to demonstrate the characteristics and identifiability of Pi 2 onsets using high latitude magnetometer array data and middle and low latitude normal magnetograms. We shall particularly emphasize the localized character of these micropulsation bursts.

To demonstrate the characteristics of Pi 2 pulsations, we shall utilize two suites of data all of which were recorded by the magnetometer arrays operated in northwestern Canada by the University of Alberta:

1) Meridian line data: In this configuration nine stations were arrayed along a common geomagnetic meridian $\left(\sim 300^{\circ} \mathrm{E}\right)$ in $1971-72$.

2) Cross array: In this configuration, four stations lay along a common meridian $\left(\sim 300^{\circ} \mathrm{E}\right)$ and three lay along a common line of geomagnetic latitude $\left(\sim 67.5^{\circ} \mathrm{N}\right)$.

Each station in each array was equipped with a three-component fluxgate magnetometer oriented in the $(H, D, Z)$ coordinate system. The data were recorded directly in digital form on magnetic tape with a sample spacing of $1.92 \mathrm{sec}$ after low pass filtering.

\section{Development of Pi 2 Activity as a Function of Latitude}

The development of magnetospheric substorm has been the topic of intense study since AKASOFU (1964) proposed the presently accepted development scheme of the auroral substorm. The development is described as it proceeds by two phases: an expansion phase where arcs in the midnight sector of the auroral oval brighten and the region of brightened auroral forms expands poleward leaving behind a region of irregular auroral bands and forms. This is followed by a recovery phase, in which the irregular auroral forms gradually reconfigure into east-west aligned arcs. These arcs gradually shift equatorward until the disturbed sector returns to its pre-substorm configuration. In addition the westward edge of the substorm disturbed region, characterized by the westward travelling surge expands into the evening sector poleward of the northern edge of the diffuse auroral oval. The substorm disturbed region is generally the site of westward ionospheric current whose magnetic signature is intense negative $H$-component bays as observed on the ground under the disturbed region.

In recent years, it has become clear that the poleward expansion of the auroral arcs is not steady, but occurs in discrete jumps. Each poleward jump signifies the appearance of discrete auroral forms and a filamentary westward electrojet poleward of the pre-existing substorm disturbed region (KISABETH and RosTOKER, 1971, 1974). This step-wise development of the electrojet is significant in that it suggests that rapid changes in magnetospheric current flow can only involve currents of limited scale size. In addition sudden changes in any electrical circuit will often result in a ringing phenomenon, and Boström (1972) has, in fact, attributed Pi 2 oscillations to the ringing of the substorm current configuration. In this context we may look at Pi 2 development as the substorm electrojet expands poleward, in the manner expounded by OLSON and ROSTOKER (1975). They show an event, for which the magnetograms are shown in Fig. 2 and the filtered micropulsation records in Fig. 3. 

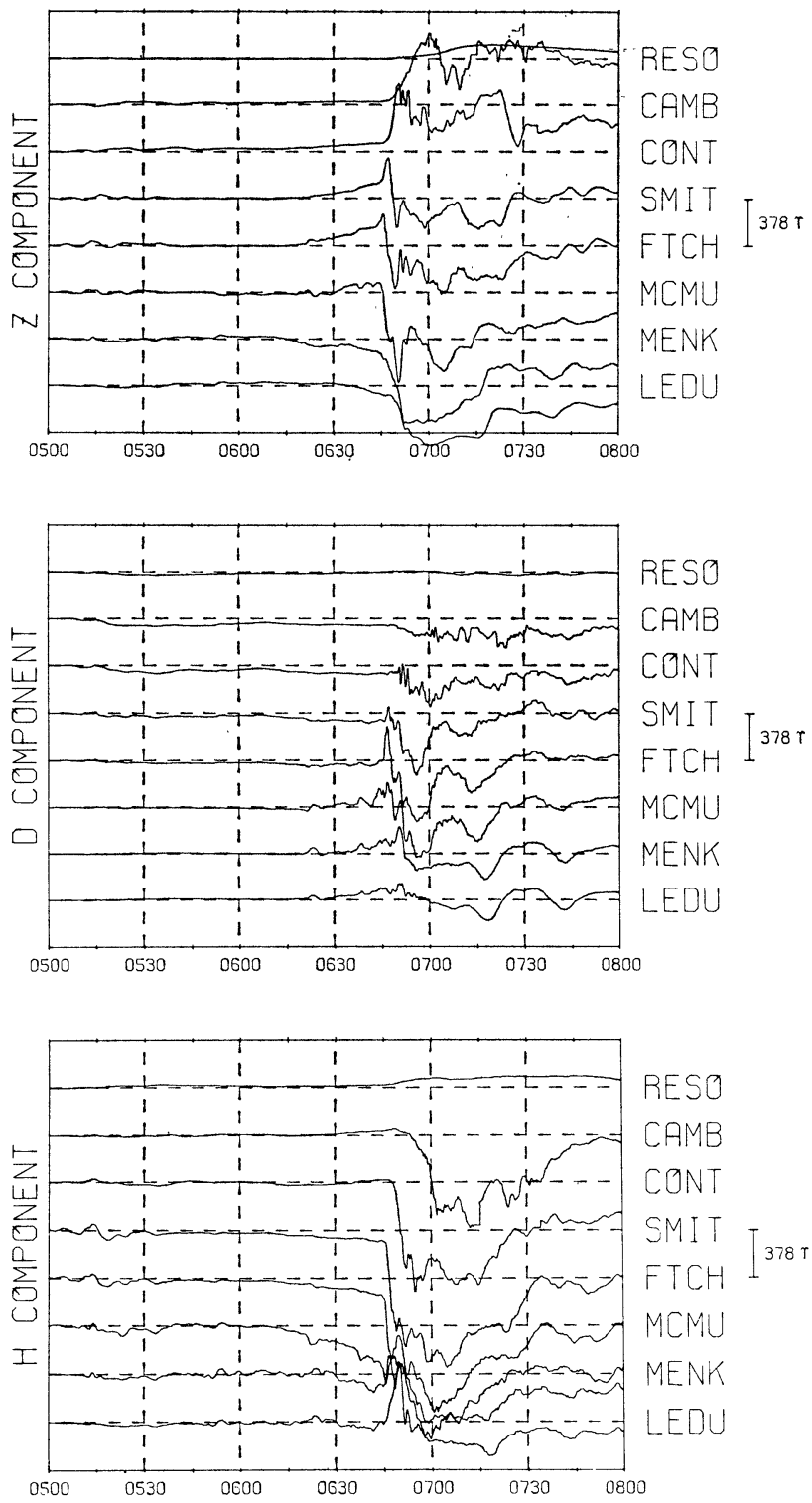

Fig. 2. Magnetograms from the University of Alberta magnetometer chain for the interval 0500-0800 UT on November 23, 1971. The stations are arranged with the northernmost station (RESO, $83^{\circ} \mathrm{N}$ ) at the top of the frame and (LEDU, $60.9^{\circ} \mathrm{N}$ ) at the bottom of the frame. Note the progressive delay in onset times in the high latitude stations. 

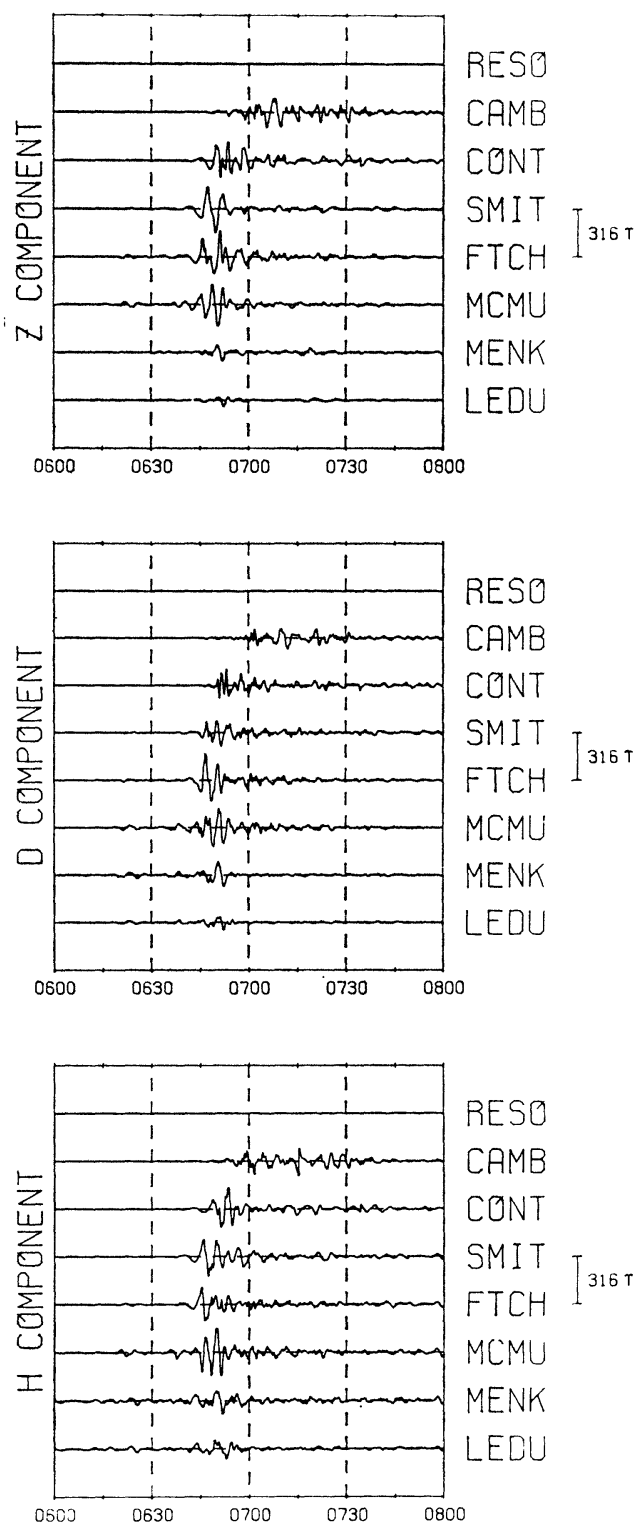

Fig. 3. Band pass filtered data for the November 23, 1971 event. The filter corner frequencies are 1.0 and $20 \mathrm{mHz}$.

The $Z$-component of the micropulsation is a good indicator of the presence of the source of pulsations over the observing stations. Figure 4 shows that the onset of Pi 2 pulsations on the $Z$-component signals the arrival of the poleward portion of the electrojet over the observing station. Thus, at auroral zone stations, monitoring the $Z$-component of the Pi 2 allows one to detect when the poleward border of the electrojet has expanded over the observing station. 


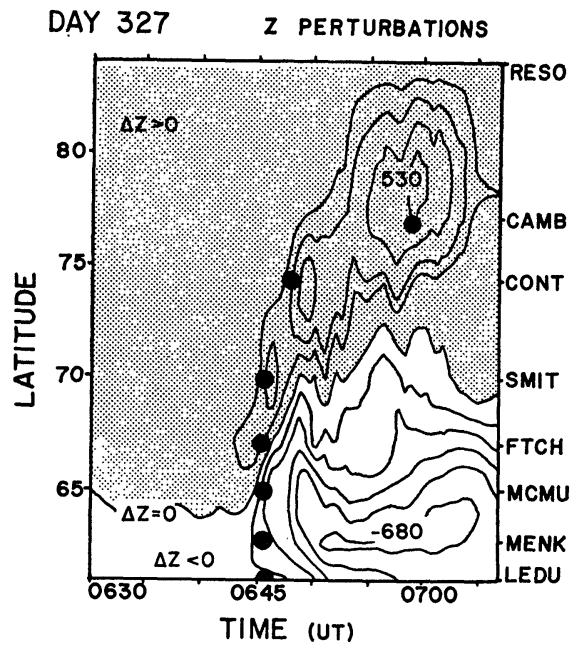

Fig. 4. Contour diagram of the $Z$-component perturbations during the November 23, 1971 event (after Olson and Rostoker, 1975). The diagram shows the intensity of the $Z$ perturbations, which indicate the presence of overhead currents, as a function of latitude and local time. Extrema in the $Z$ perturbation levels delineate the approximate boundaries of the electrojet at a given instant. The solid dots show the onset times of Pi 2 pulsations at each station in the chain.

From Fig. 3 it can be seen, comparing the SMIT, CONT and CAMB records that the clearly identifiable onsets in all components occur at different times. This is because of the large signal enhancement when the electrojet is overhead compared to when it is not. Thus it is clear that clearly defined Pi 2 enhancement can only be associated with substorm intensifications and not necessarily with the onset of substorm sequences. In addition, low level Pi 2 activity at one location may well (and probably does) indicate strong Pi 2 activity at some distance from the observing point.

\section{Development of Pi 2 Activity as a Function of Longitude}

In the remaining portion of this paper we shall present a detailed analysis of the magnetic activity which occurred on August 29, 1974. In this analysis the use of low latitude standard magnetograms along with auroral zone records from the University of Alberta magnetometer array will allow us to observe the progress of a series of substorm intensifications and their associated Pi 2 wave trains across North America. Similar analyses have been performed on data from other days with very similar results.

Figure 5, which shows the $H$-component records from various low latitude magnetograms, will allow us to set the scene for the event. The stations are arranged on the figure with the easternmost station (JUAN) at the top and the westernmost at the bottom. We direct your attention to the following features: 


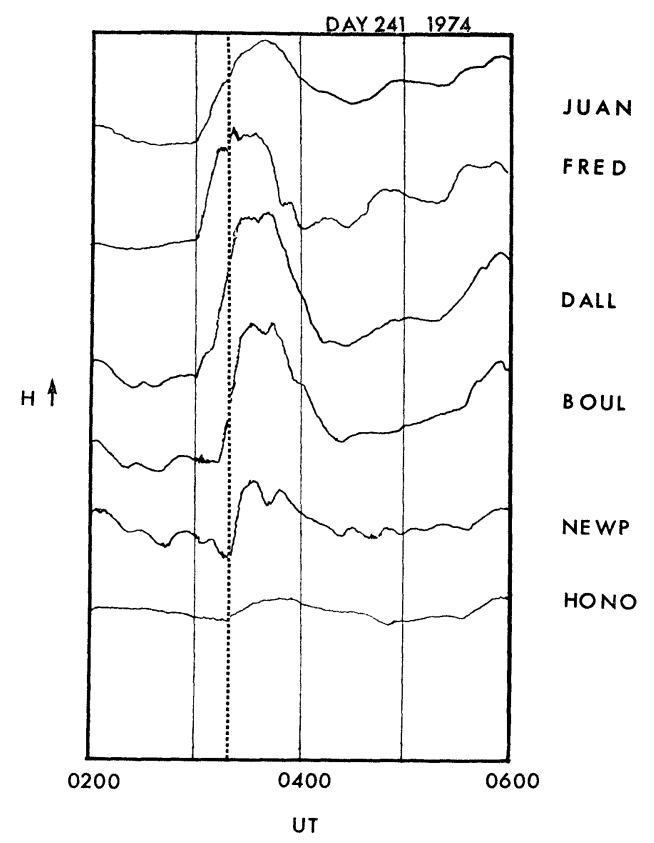

Fig. 5. $H$-component data for August 29, 1974 from low latitude stations at San Juan, Fredericksburg, Dallas, Boulder, Newport and Honolulu in the North American sector. The station data are arranged sequentially from east to west with the easternmost station data at the top. Substorm onsets may be seen at 0300, 0310, 0315 and 0320 UT. The vertical dashed line marks the 0320 UT event.

1) A clear substorm onset is observed at 0300 UT from JUAN to DALL. Note that the associated Pi 2 at BOUL does not show peak amplitude at the moment of onset. The maximum occurs 3 to 4 min after the hour. The use of this station's Pi 2 would result in an error in timing the moment of substorm onset.

2) A second onset occurs at 0310 UT. The signature is clear at DALL. The high latitude data from Fort Churchill (not shown here) showed a sudden negative excursion in $\Delta H$ at this time.

3) A third onset occurred near 0315 UT. This onset is clearest at BOUL and an associated Pi 2 is present at FRED. In the auroral oval the onset was masked by activity associated with the $0310 \mathrm{UT}$ onset.

4) The fourth onset which signaled the start of major activity in the western sector of North America occurred at $0320 \mathrm{UT}$ as indicated by the dashed line on the figure. It is clearly evident at NEWP and shows in the HONO record. The breadth of this region indicates a large scale event. $\Delta H$ variations are positive across the entire continent and into the mid-Pacific Ocean region.

Although there is evidence for further activity in this substorm sequence we shall limit our study to these four events, because of the lack of station coverage in the east Pacific area. Thus in these data we may observe the passage of substorm 
activity across North America in four intensifications. We will now focus on the data from our array in the Alberta sector for a detailed view of the activity seen from one sector.

Figure 6 shows the high latitude magnetograms from the University of Alberta magnetometer array. The figure shows three panels which contain the $H, D$ and $Z$ component variations from the array. At the top of each panel is URAN, the easternmost station. SMIT and HAYR lie at the same latitudes as URAN $\left(\sim 67.5^{\circ} \mathrm{N}\right)$ with HAYR some $10^{\circ}$ west of URAN. MCMU lies approximately $3^{\circ}$ south of SMIT
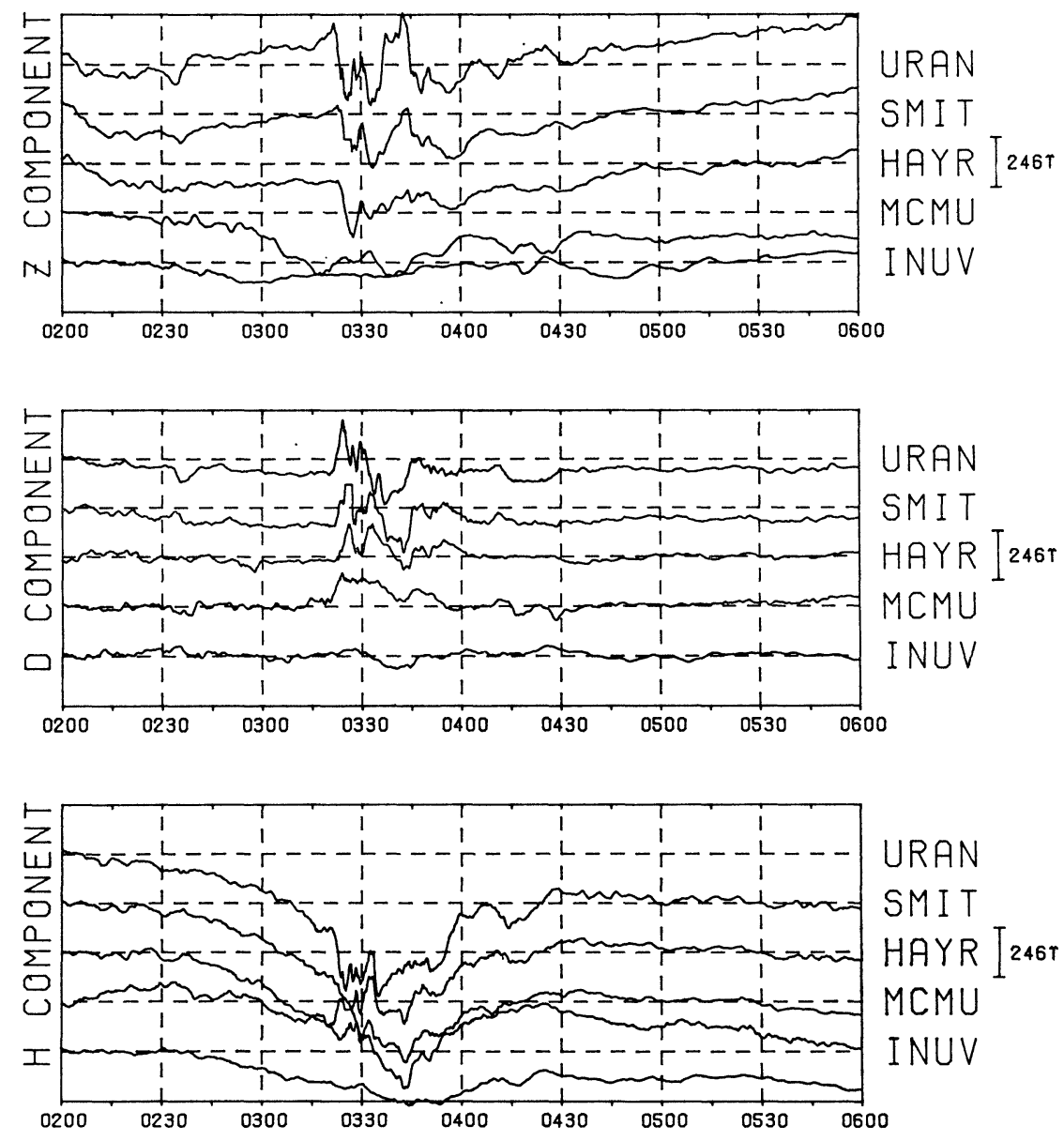

Fig. 6. Magnetic records from the University of Alberta array showing the high latitude records for the August 29, 1974 event. The principal substorm onset in this sector occurred at 0320 and can be seen in all components. There is also evidence of the 0315 onset which occurred to the east of the Alberta sector. 
on the same meridian. INUV lies to the north and far to the west of the other stations.

Recall that we observed onsets at 0300, 0310, 0315 and 0320 UT in the low latitude magnetograms. Here, on this scale, we see no clear evidence of the $0300 \mathrm{UT}$ onset. The first indication of a change in activity occurs at 0315 UT where URAN, at the eastern edge of the array sees a noticeable $\Delta Z$ inflection. The other stations do not show this so clearly but they begin to change slightly in accordance with their position relative to the center of activity. The 0320 UT event impacts the entire array and the large $+\Delta D$ signals the arrival of the westward travelling surge over the station array. Upon careful analysis, the $\Delta D$ maximum and the onset of the major inflection can be seen to occur at 0321 UT at URAN and 0322 UT and SMIT reflecting the rapid westward expansion $(\sim 3 \mathrm{~km} / \mathrm{sec})$ within the sector disturbed by the $0320 \mathrm{UT}$ onset. As mentioned earlier there is continuing activity after $0320 \mathrm{UT}$ with a rather clear intensification of the electrojet near 0330 UT being noticeable in the Alberta array.

Thus the sequence of onsets at 0300, 0310, 0315 and $0320 \mathrm{UT}$ is established in both low latitude and auroral zone records. Now let us turn to the Pi 2 content of these records. The observation of Pi 2 activity on normal magnetograms is often difficult because of the near simultaneity of the pulsation and associated bay. This causes the pulsation to ride on very steep ramps and become lost in the line thickness. Further the sensitivities of the standard magnetograms varies from high at low latitudes to low at high latitudes making a quantitative threshold for pulsation activity difficult to establish. We may avoid these difficulties by turning to an analysis of the digital data from the University of Alberta array. For brevity we shall treat the data from URAN with the comment that the analysis of data from the other stations showed similar features.

Figure 7 a shows in the upper panel a sonogram derived from the $H$-component data series in the lower panel. The data span the time interval 0245-0350 UT. The sonogram is constructed from overlapping data samples each of which is 4 min and $6 \mathrm{sec}$ long. The samples overlap by half their length. Thus every other spectrum, represented by a vertical column in the array, is an independent spectrum. The result is a smoothing in the time axis of approximately $4 \mathrm{~min}$. The individual spectra (vertical columns) are characterized by 4 degrees of freedom and digitized in $10 \mathrm{db}$ steps. The spectra have been smoothed using a $10 \mathrm{mHz}$ window. We shall find that onsets delineated above are most clearly visible in the $25-50 \mathrm{sec}$ period band. There is also evidence of the onsets in the long period $(T>100)$ band. Here we have graphic evidence of the difficulty of detecting unique Pi 2 signals in auroral zone data. The nominal Pi 2 band, between 40 and $150 \mathrm{sec}$, displays a steady background level which raises the threshold for Pi 2 detection in that period range. The Pi 1 band provides a greater contrast in signal to noise and is more useful in this instance.

In the first sonogram, data from the $H$-component at URAN are shown. In this figure the onsets at 0300 and 0320 UT are clearly visible as broad band short duration bursts. The onsets at 0310 and $0315 \mathrm{UT}$ are not resolved in the 25 to $50 \mathrm{sec}$ band 
due to contamination of the spectra by low frequency noise and the width of the data window. Nevertheless the long period $(T>100 \mathrm{sec})$ portion of the spectrum shows separate enhancements above the background at 0310 and 0315 UT. Note as well the continuing activity after 0320 UT.
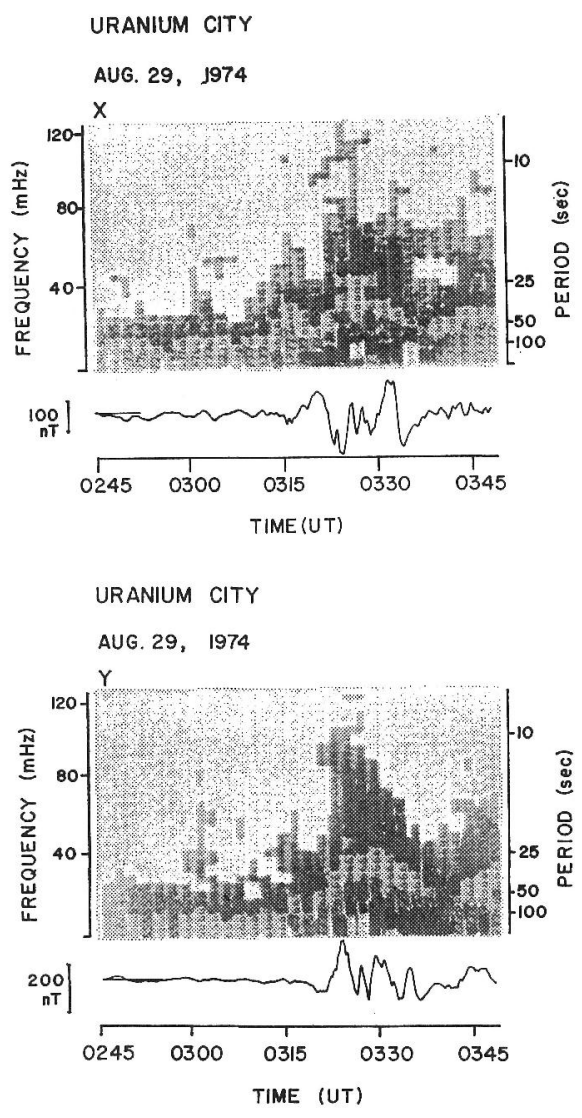

URANIUM CITY

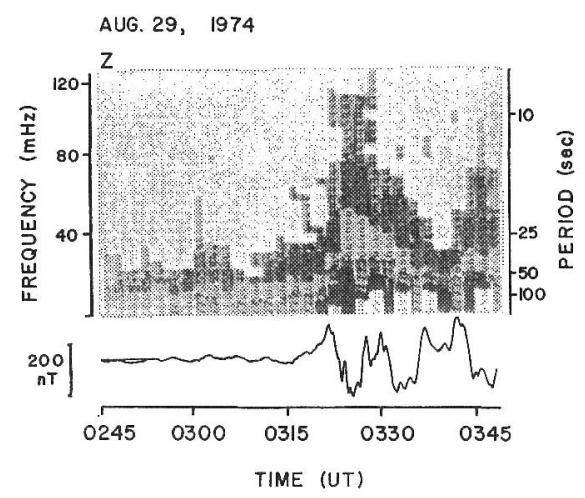

Fig. 7a. A sonogram of the $H$-component data at Uranium City covering 0245-0345 UT on August 29, 1974. The 0300, 0310, 0315 and 0320 UT onsets are most clearly seen in the Pi 1 band where there is good signal contrast. See text for analysis details.
Fig. 7b. A sonogram of the Uranium City $D$ component data covering the interval 0245 0345 UT on August 29, 1974. Onsets at 0300, 0310,0315 and $0320 \mathrm{UT}$ are clearest again in the Pi 1 band.

Fig. 7c. A sonogram of the Uranium City $Z$ component data covering the interval 02450345 UT on August 29, 1974. Here the 0300, 0315 and 0320 UT onsets may be seen. See text for details. 
Figure $7 \mathrm{~b}$ shows a similar sonogram for the $D$-component at URAN. Again the 0300 and 0320 UT onsets are clearly defined. The 0315 UT onset is also visible in the sonogram even though it is somewhat obscured by background activity. The identification of an onset at $0310 \mathrm{UT}$ from these data alone appears somewhat more tenuous, although there is some suggestion of additional activity near that time.

The final sonogram presented in Fig. 7c shows the $Z$-component activity. While these data are quieter than the $H$ - and $D$-component data one can still see the onsets at 0300,0315 and $0320 \mathrm{UT}$. The $0310 \mathrm{UT}$ onset is not visible here. It is lost in the general background noise in the Pi 2 band.

We may summarize our results by noting that when we use Pi 2 onsets to identify substorm onsets or intensifications, we encounter basic limitations in the mixing of wave trains (viz. a Pi 2 onset occurs before a prior Pi 2 dies out) and background noise in the Pi 2 range (particularly at high latitude stations). On normal magnetograph recordings the wave trains mix with one another making determination of onsets often difficult. For sonograms the width of the spectral windows causes uncertainty in the estimation of the onset time and often increases in power are difficult to extract from the power associated with background noise. We conclude by noting that the sonogram, when used as we have above, represents a pulsation detector based upon changes of energy density in certain spectral bands. Clearly it is of marginal use for short-lived signals in a noisy environment.

\section{Conclusions}

We finish our discussion of the use of Pi micropulsations in identifying substorm onsets by summarizing some of the key points made in the foregoing text.

As regards middle and low latitude observatories we may say the following:

i) Pi 2 onsets are observable over 1-2 time zones under average circumstances. However, sometimes the amplitudes are low or the waveform is not easily recognizable as a Pi 2 according to existing criteria. In addition the mixing of consecutive wave trains may make some onsets difficult to define.

ii) For normal magnetograms, Pi 2 wave trains may have their structure masked as they ride on a ramp associated with the accompanying geomagnetic bay.

iii) Pi 2 amplitude extrema may not coincide with a substorm onset. (That is, one should not demand an initial large amplitude pulse at the beginning of a wave train as a necessary condition for classification of that wave train as a Pi 2.) This is because the wave train may be shaped by transmission processes at the signal propagates from the source to the field point.

As regards auroral oval observatories, we may say the following:

i) Pi 2 "onsets" occur as the substorm electrojet poleward border passes over the observing station.

ii) The Pi frequency band is often contaminated with local electrojet noise, making the identification of distant onsets sometimes difficult. This problem would be accentuated as the general level of magnetospheric activity increases. 
In conclusion, if $\mathrm{Pi} 2$ micropulsations are to be used in the future for the identification of substorm onsets and/or intensifications we recommend that:

a) Agreement be reached on the character of wave trains which uniquely define them as Pi 2.

b) That onsets not be identified solely by the Pi 2 technique. Any individual event should be studied using multistation data and, for each station, all three components of the perturbation field should be considered.

This research was supported by the National Research Council of Canada.

\section{REFERENCES}

Akasofu, S.-I., The development of the auroral substorm, Planet. Space Sci., 12, 273-282, 1964.

Akasofu, S.-I., Polar and Magnetospheric Substorms, Springer-Verlag, New York, 1968.

ANGenheister, B., Über die Fortpflanzungs-Geschwindigkeit magnetischer Storungen und Pulsationen, Bericht über die Erdmagnetischen Cheltenham und Tsingtau in September 1911, Goettingen Nachr. Ges. Wiss., 1912.

Bоsтröm, R., Magnetosphere-ionosphere coupling, in Critical Problems of Magnetospheric Physics, edited by E.R. Dyer, Jr., pp. 139-156, IUCSTP Secretariat, Washington, D.C., 1972.

Jacobs, J.A., Y. Kato, S. MAtsushita, and V.A. Trortskaya, Classification of geomagnetic micropulsations, J. Geophys. Res., 69, 180-181, 1964.

Kisabeth, J.L. and G. Rostoker, Development of the polar electrojet during polar magnetic substorms, J. Geophys. Res., 76, 6815-6828, 1971.

KisAbeth, J.L. and G. Rostoker, The expansive phase of magnetospheric substorms. 1. Development of the auroral electrojets and auroral arc configuration during a substorm, J. Geophys. Res., 79, 972-984, 1974.

Fununishi, H. and T. Hirasawa, Progressive change in Pi 2 power spectra with the development of magnetospheric substorms, Rep. Ionos. Space Res. Japan, 24, 45-65, 1970.

Olson, J.V. and G. Rostoker, Pi 2 pulsations and the auroral electrojet, Planet. Space Sci., 73, 1129$1139,1975$.

Pytte, T., R.L. McPherron, E.W. Hones, Jr., and H.I. West, Jr., Multiple satellite studies of magnetospheric substorms: Absence of substorm-onset signatures during prolonged auroral-zone bay activity, J. Geophys. Res., 83, 663-679, 1978.

Rostoker, G., Macrostructure of geomagnetic bays, J. Geophys. Res., 73, 4217-4229, 1968.

SAIto, T., Pi 3-type polar magnetic variation due to a fluctuating three-dimensional current system during a magnetospheric substorm, IAGA Bulletin, No. 36, 1976.

SAito, T. and K. Yumoto, Comparison of the two-snake model with the observed polarization of the substorm-associated magnetic pulsation Ps 6, J. Geomag. Geoelectr., 30, 39-54, 1978.

Saito, T., K. Yumoto, and Y. Koyama, Magnetic pulsation Pi 2 as a sensitive indicator of magnetospheric substorm, Planet. Space Sci., 24, 1025-1029, 1976a.

Saito, T., T. Sakural, and Y. Koyama, Mechanism of association between Pi 2 pulsation and magnetospheric substorm, J. Atmos. Terr. Phys., 38, 1265-1277, 1976b.

Sakurai, T. and T. Sarto, Magnetic pulsation Pi 2 and substorm onset, Planet. Space Sci., 24, 573$575,1976$.

Sergeev, V.A., On the longitudinal localization of the substorm active region and its changes during the substorm, Planet. Space Sci., 22, 1341-1343, 1974.

WIENs, R.G. and G. Rostoker, Characteristics of the development of the westward electrojet during the expansive phase of magnetospheric substorms, J. Geophys. Res., 80, 2109-2128, 1975. 